\title{
Die Zeitpräferenz von Landwirten
}

\author{
- Ein experimenteller Methodenvergleich
}
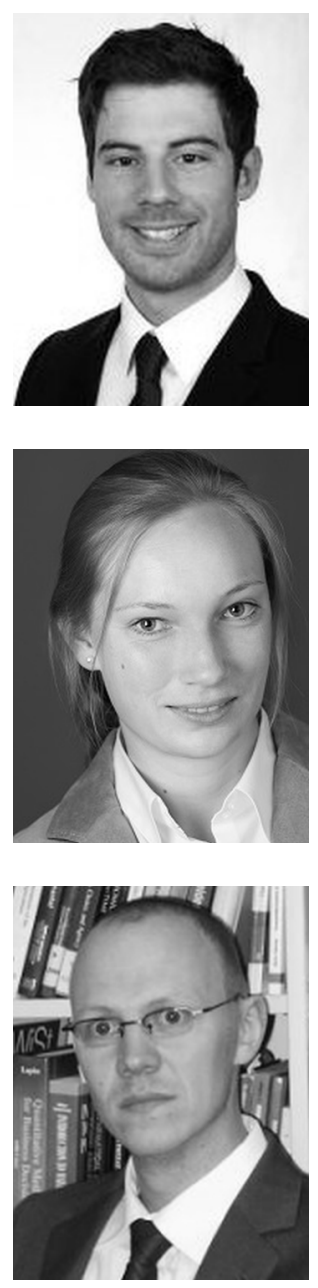

\section{Einleitung}

In vielen Situationen müssen Entscheidungsträger zwischen Alternativen wählen, die nicht nur Konsequenzen in der Gegenwart, sondern auch für die Zukunft mit sich bringen. Beispielsweise müssen bei Investitionsvorhaben die unsicheren zukünftigen Rückflüsse mit den in der Gegenwart anfallenden Investitionskosten abgewogen werden (Ablbrecht/Weber 1997). Dabei bestimmt die persönliche Zeitpräferenz einer Person deren intertemporales Austauschverhältnis zwischen heutigem und zukünftigem Konsum (Anderhub et al. 2001; Frederick 2003, 89f.). Das bedeutet, dass die Zeitpräferenz anzeigt, wie viel Personen dafür fordern, ihren Konsum von einem

\footnotetext{
* Korrespondenzautor.
} 
zeitlich näheren auf einen zeitlich entfernteren Zeitpunkt zu verschieben. Sobald Kosten oder Nutzen zu unterschiedlichen Zeitpunkten anfallen, ist die Zeitpräferenz ein massgeblicher Bestimmungsfaktor in der Entscheidungsfindung (Harrison et al. 2002).

Die Untersuchung der Zeitpräferenz ist sowohl für die individuelle betriebliche Entscheidungsfindung und Beratung, als auch zur Unterstützung von politischen Entscheidungen von grosser Bedeutung (Anderson/Stafford 2009; Laury et al. 2012). Gollier/Zeckhauser (2005) stellen heraus, dass die Zeitpräferenz als Bestimmungsfaktor für das Spar- und Investitionsverhalten die wichtigsten Entscheidungen ökonomischer Akteure beeinflusst. Laury et al. (2012) beschreiben die Zeitpräferenz als wichtigen Faktor bei der Bestimmung der Ausbildungsdauer. Mit dem Erwerb eines höheren Ausbildungsgrads kann in Zukunft ein höheres Einkommen erzielt werden, welches zu höherem Konsum verwendet werden kann. Während der Ausbildungszeit muss aber auf Einkommen (Konsum) verzichtet werden. Im gesamtgesellschaftlichen Kontext ist die soziale Diskontrate ebenfalls von entscheidender Bedeutung. Diese wird als Kalkulationsbestandteil in vielen öffentlichen Investitionsrechnungen verwendet, so beispielsweise in der deutschen Wegekostenrechnung (Link 2003), der Berechnung zur Erweiterung von Stromnetzen (Friedl et al. 2014) oder bei Entscheidungen zu Massnahmen im Rahmen der Klimapolitik (Stern 2006).

Aufgrund der dargestellten Relevanz der Zeitpräferenz für unternehmerische Entscheidungen wird diese in vielen wissenschaftlichen Arbeiten untersucht (Ablbrecht/Weber 1997; Coble/Lusk 2010; Andreoni/Sprenger 2012). Die Quantifizierung der Zeitpräferenz erfolgt mittels der Bestimmung von Diskontraten (Coller/Williams 1999; Andersen et al. 2008; Benhabib et al. 2010; Bocquého et al. 2013). Dazu werden verschiedene methodische Vorgehensweisen genutzt. Die Ermittlung der Diskontraten kann mittels felddatenbasierter statistisch-ökonometrischer Ansätze (Lence 2000) oder mittels experimentell gewonnener Daten erfolgen (Pender 1996; Duquette et al. 2012; Bocquého et al. 2013). Basierend auf der nutzentheoretischen Fundierung der Zeitpräferenz können mit statistischökonometrischen Methoden Diskontraten aus verschiedenen Felddatensätzen errechnet werden. So verwendet Hausman (1979) Daten zum Kauf und der Nutzung von elektrischen Gebrauchsgütern zur ökonometrischen Berechnung der Diskontrate und ermittelt dabei eine individuelle Diskontrate von etwa 20\%. Moore/Viscusi (1990) verwenden fünf unterschiedliche ökonometrische Vorgehensweisen zur Diskontratenbestimmung und wenden diese auf Arbeitsmarktdaten an. Sie ermitteln Diskontraten von 1\% bis 14\%. Lence (2000) ermittelt die Risikoeinstellung und die Zeitpräferenz von Landwirten mit Hilfe eines generalisierten Erwartungsnutzenmodells anhand von Felddaten und berechnet Diskontraten von 3\% bis 5\%. Zusammenfassende Überblicke zu Diskontraten aus verschiedenen Studien bzw. mit verschiedenen Methoden geben unter anderem Train (1985), Moore/Viscusi (1990) und Frederick et al. (2002).

Neben den ökonometrischen Methoden zur Diskontratenbestimmung auf Basis von Felddaten wird diese experimentell erfasst. Das ist beispielsweise darin begründet, dass Felddaten häufig aggregiert vorliegen, was eine ökonometrische Diskontratenbestimmung erschwert. Zudem besitzen Experimente weitere Vorteile gegenüber der felddatenbasierten Erfassung: So erfolgt die Datenerhebung in Experimenten unter kontrollierten Bedingungen (Smith 1982). Dadurch kann die Diskontrate erfasst werden, ohne dass beispielsweise finanzielle Restriktionen eine Rolle spielen, was bei Felddaten nicht kontrolliert werden kann. Ausserdem können in Experimenten weitere Daten (z. B. das Alter der Entscheider) erfasst werden, die aus Felddaten nicht hervorgehen. Dies ermöglicht die Ableitung kausa- 
ler Zusammenhänge und steigert die interne Validität der Ergebnisse (Roe/Just 2009). Auch die externe Validität der experimentell erhobenen Daten wurde im Bezug auf die Diskontratenerfassung überprüft und bekräftigt (Chabris et al. 2008). Bei der Anwendung verschiedener experimenteller Methoden zur Quantifizierung der Zeitpräferenz wurden unterschiedlich hohe Diskontraten (in einem Spektrum von minus 6\% bis annähernd unendlich) für vergleichbare Personengruppen und Individuen festgestellt (Frederick et al. 2002).

Um die Bedeutung der Verwendung einer zutreffenden Diskontrate zu veranschaulichen, wird ein Zahlenbeispiel verwendet: Es sei die Möglichkeit gegeben, eine Investition durchzuführen, die mit unmittelbaren Kosten von $75 €$ verbunden ist. Nach zwei Jahren liefert diese Investition eine Rückzahlung in Höhe von $85 €$ und es werden Diskontraten von 5\% und $10 \%$ angenommen. Demnach wäre der Barwert der Investition $85 € /(1,05)^{2}=77,10 €$ bei einer Diskontrate von $5 \%$ und $70,25 €\left(85 € /(1,10)^{2}\right)$ bei einer Diskontrate von $10 \%$. Im Falle einer Diskontrate von 5\% übersteigt der Barwert der Investition die Investitionskosten. Im Gegensatz dazu ist im Falle einer Diskontrate von 10\% - und damit einer höheren Gegenwartspräferenz des Konsums - der Barwert kleiner als die Investitionskosten.

Die Unterschiede in der gemessenen Diskontratenhöhe gehen unter anderem auf den „magnitude effect", also die Verwendung unterschiedlich hoher Geldbeträge bei der Erfassung der Diskontrate, zurück (Pender 1996; Frederick et al. 2002; Bocquébo et al. 2013). Auch das explizite Ausweisen des Zinssatzes in den Experimenten beeinflusst die Höhe der ermittelten Diskontraten (Coller/Williams 1999). Andersen et al. (2008) geben zudem an, dass es zu einer fehlerhaften Bestimmung der Diskontraten kommen kann, wenn a priori von risikoneutralen Entscheidern ausgegangen wird (Holt/Laury 2002; Andersen et al. 2006; Coble/Lusk 2010). Daher erfassen Andersen et al. (2008) die Diskontraten nach der Methode von Coller/Williams (1999; CW-task) sowie die Risikoeinstellung nach Holt/ Laury (2002; HL-task) und korrigieren die individuelle Diskontrate mit der Risikoeinstellung der Experimentteilnehmer (CW/HL-task). Eine weitere experimentelle Methode zur Ermittlung von Diskontraten wird von Laury et al. (2012) vorgestellt (L-task). Hierbei ist es nicht erforderlich die Risikoeinstellung der Teilnehmer separat zu erfassen. Die Diskontrate wird mit einer einzigen experimentellen Aufgabenstellung festgestellt und die Risikoeinstellung implizit erfasst. Die Erhebung der individuellen Diskontraten wird dadurch deutlich vereinfacht und mögliche Fehlerquellen können vermieden werden. Die L-task wurde, wie in ökonomischen Experimenten üblich (Coller/Williams 1999; Anderhub et al. 2001; Anderson/Stafford 2009; Coble/Lusk 2010), zur Messung der Diskontraten von Studierenden angewendet. Dabei erfassen Laury et al. (2012) die Diskontraten der Studierenden mit der L-task und der CW/HL-task, wobei keine unterschiedlichen Ergebnisse festgestellt werden können.

Ziel dieses Beitrags ist es zu klären, ob die CW/HL-task und die L-task, bei der Anwendung mit Landwirten, zu gleichen Ergebnissen führen. Ausserdem soll die Sensibilität der beiden Methoden gegenüber experimentellen Designänderungen festgestellt werden. Die beiden Methoden sind von besonderem Interesse, da die CW/HL-task eine häufig angewendete und die L-task eine neue, vereinfachende Erfassungsmethode darstellt. Aufgrund der grossen Schwierigkeiten bei der Übertragbarkeit des Verhaltens von Studierenden auf Unternehmer (Barr/Hitt 1986; Maart-Noelck/Mußhoff 2014), werden die Diskontraten von Unternehmern erhoben. Der Fokus auf Landwirte ist insbesondere deshalb relevant, da Landwirte in ihrem Berufsalltag vielen Risiken ausgesetzt sind. So spielen neben dem 
Wetterrisiko auch der Befall von Pflanzen durch Krankheiten oder Schaderreger, Preisrisiken, technologische sowie politische Risiken eine bedeutende Rolle (Moschini/Hennessy 2001; Herberich/List 2012). Des Weiteren treffen Landwirte Entscheidungen mit langer Fristigkeit und mit einem hohen Anteil versunkener Kosten (z. B. der Anbau mehrjähriger Kulturen oder der Bau von verwendungsspezifischen Stallgebäuden) (Lambson/Jensen 1995). Bisher wurden Diskontraten von Landwirten vorwiegend ohne die Berücksichtigung der Risikoeinstellung gemessen (Duquette et al. 2012; Bocquého et al. 2013).

Die bestehende Literatur wird mit diesem Beitrag in vier Punkten erweitert: Erstens werden risikoadjustierte Diskontraten von Unternehmern, genauer von Landwirten, durch die Anwendung der CW/HL-task und der L-task bestimmt und die Ergebnisse beider Methoden miteinander verglichen. Dieser Vergleich der beiden Methoden wurde bislang ausschliesslich mit Studierenden durchgeführt. Zweitens wird überprüft, ob eine Veränderung der Höhe der zur Erfassung der Diskontrate verwendeten Geldbeträge in der CW/HL-task und der L-task bei Landwirten zu abweichenden Ergebnissen führt. Drittens wird überprüft, ob die Darstellung des Zinssatzes im Experiment einen Einfluss auf die mit der CW/HL-task erfasste individuelle Diskontrate hat. Viertens wird der Einfluss soziodemografischer und betriebsspezifischer Faktoren auf die risikoangepassten Diskontraten von Landwirten untersucht.

Im folgenden Abschnitt 2 werden Hypothesen aus der bestehenden Literatur hergeleitet. Die Beschreibung der methodischen Vorgehensweise erfolgt in Abschnitt 3. In Abschnitt 4 werden die deskriptive Statistik und die Methode zur Datenauswertung dargestellt, um anschliessend in Abschnitt 5 die aufgestellten Hypothesen zu überprüfen. Der Beitrag schliesst mit Schlussfolgerungen und einem Ausblick in Abschnitt 6.

\section{Hypothesen}

Die L-task wurde bisher nur auf Studierende angewendet. Bei ihrer Untersuchung ermitteln Laury et al. (2012) auch die Diskontraten mit der CW/HL-task und vergleichen die Ergebnisse beider Methoden. Es zeigt sich, dass die Anwendung der beiden Methoden bei Studierenden zu vergleichbaren Diskontraten führt. Eine Übertragung dieses Methodenvergleichs und der Diskontraten von Studierenden auf Unternehmer ist jedoch nicht ohne Weiteres möglich. Barr/Hitt (1986) erläutern, dass die Aussagekraft von Experimenten mit Studierenden in der Verhaltensforschung umstritten ist und zeigen, dass sich Manager bei Auswahlentscheidungen systematisch anders verhalten als Studierende. Eine mögliche Begründung dafür geben Andersen et al. (2010), die die Gruppe der Studierenden im Vergleich zu Unternehmern in Merkmalen wie dem Alter und dem Bildungsniveau als homogener beschreiben. Harrison/List (2008) und Khera/Benson (1970) warnen aufgrund unterschiedlicher Erfahrungen von Unternehmern und Studierenden hinsichtlich der Führung von Unternehmen davor, das Verhalten von Studierenden zu generalisieren. Im landwirtschaftlichen Kontext zeigen Maart-Noelck/Mußhoff (2014), dass das Entscheidungsverhalten von Studierenden nicht auf Landwirte übertragbar ist. Damit lässt sich festhalten, dass eine Übertragung der Ergebnisse von Experimenten mit Studierenden auf Unternehmer generell schwierig ist. Da ein Vergleich der Diskontraten nach L-task und CW/HL-task bislang nicht mit Landwirten, sondern mit Studierenden durchgeführt wurde, werden die individuellen Diskontraten beider Methoden verglichen.

Bei der L-task werden die Zinssätze zur Bestimmung der Diskontraten bewusst nicht angezeigt, um die Experimentteilnehmer bei ihren Entscheidungen nicht zu beeinflussen. In 
der CW-task werden die Zinssätze angezeigt. Laury et al. (2012) können bei Studierenden keine Unterschiede in den Ergebnissen der Methoden mit und ohne Angaben von Zinssätzen feststellen. Coller/Williams (1999) zeigen allerdings, dass die zusätzliche Angabe des Jahreszinses, mit dem die heutigen Zahlungen bis zum zukünftigen Auszahlungszeitpunkt aufgezinst werden, bei der experimentellen Messung der Zeitpräferenz einen signifikanten Einfluss hat: Das explizite Ausweisen des Jahreszinses im Experiment führt ceteris paribus zu niedrigeren Diskontraten. Basierend auf diesen Erkenntnissen werden die folgenden Hypothesen geprüft:

H1a: Bei der Anwendung mit Landwirten unterscheiden sich die Ergebnisse einer individuellen Zeitpräferenzmessung in der CW/HL-task und der L-task nicht.

H1b: Wenn bei der CW/HL-task die Diskontierungssätze nicht explizit ausgewiesen werden, unterscheiden sich die Diskontraten zwischen beiden Methoden.

Bei der experimentellen Ermittlung der Diskontraten von Studierenden durch Benzion et al. (1989) und durch Thaler (1981), aber auch von Landwirten und Landarbeitern durch Pender (1996), konnte ein Effekt der Höhe der zur Erfassung verwendeten Geldbeträge festgestellt werden. Dieser Effekt wird in der Literatur als „magnitude effect“ bezeichnet und besagt, dass die Diskontrate mit steigenden Beträgen abnimmt (Frederick et al. 2002). Es wird daher der „magnitude effect“ bei Anwendung beider Methoden der Zeitpräferenzmessung auf Landwirte überprüft und folgende Hypothese untersucht:

H2: Bei Verwendung höherer Geldbeträge zur Diskontratenerfassung mit der gleichen Methode sinken die erfassten Diskontraten signifikant.

\section{Methodische Vorgehensweise}

Zur Untersuchung der aufgestellten Hypothesen wurde ein computerbasiertes Experiment entwickelt. Im folgenden Abschnitt beschreiben wir zunächst das grundsätzliche Design des Experiments. Anschliessend wird die Durchführung des Experiments beschrieben. Abschliessend wird gezeigt, wie die Diskontrate für die CW/HL-task zu berechnen ist.

\subsection{Design des Experiments}

Im Lotterie- und Auswahlteil werden im Rahmen der CW/HL-task und der L-task verschiedene Wahlentscheidungen und Lotterien durchgeführt. Zum einen werden die Diskontraten nach der CW-task ermittelt und mit der HL-task die Risikoeinstellung der Teilnehmer bestimmt, um die risikoadjustierten Diskontraten der CW/HL-task berechnen zu können. Zum anderen werden die Diskontraten mittels L-task erfasst. An den Lotterieund Auswahlteil anschliessend werden einige allgemeine Informationen zum bewirtschafteten Betrieb sowie soziodemografische Daten der Teilnehmer erhoben. Die Teilnehmer werden ausserdem gebeten, ihre persönliche Vermögenssituation im Vergleich zu anderen Betriebsleitern auf einer elfstufigen Skala einzuschätzen. Der Aufbau der Teilexperimente wird im Folgenden ausführlich beschrieben.

\subsubsection{Aufbau der CW-task zur Zeitpräferenzmessung}

In diesem Abschnitt des Experiments werden die Teilnehmer mit 20 Entscheidungssituationen konfrontiert, in denen sie jeweils zwischen einem sicheren Geldbetrag A in drei Wochen 
und einem sicheren Geldbetrag B in zwölf Wochen wählen können (vgl. Tabelle 1). Geldbetrag A liegt in allen 20 Entscheidungssituationen bei $100 €$. Geldbetrag B steigt ausgehend von $100 €$ in Entscheidungssituation 1 auf $129,48 €$ in Entscheidungssituation 20. Die nominalen und effektiven Zinssätze pro Jahr, wonach sich Geldbetrag B durch Aufzinsen in den 20 Entscheidungssituationen im Vergleich zu Geldbetrag A erhöht, werden einer Gruppe von Teilnehmern in jeder Entscheidungssituation explizit ausgewiesen. Einer zweiten Gruppe von Teilnehmern werden diese Zinssätze nicht explizit ausgewiesen. Aus dem Wechsel der Wahl von Geldbetrag A zu Geldbetrag B können die persönlichen Diskontraten der Teilnehmer - unter Annahme von Risikoneutralität - abgelesen werden. Der Vorgehensweise von Laury et al. (2012) folgend, liegen alle Auszahlungszeitpunkte der möglichen Gewinne in der Zukunft. Bei der Wahl der Zeitpunkte wird das Vorgehen von Laury et al. (2012) als Vorbild gewählt, um deren Ergebnisse möglichst gut mit den Ergebnissen dieser Untersuchung vergleichen zu können. Die Verzögerung der Auszahlung von Geldbetrag A wurde von Coller/Williams (1999) eingeführt und vermeidet den sogenannten „present bias“. Dieser bezeichnet eine starke Gegenwartspräferenz, die die Diskontrate verzerrt (Benhabib et al. 2010). Der zweite Auszahlungszeitpunkt wurde mit zwölf Wochen Verzögerung zum Tag der Experimentdurchführung gewählt, um die Transaktionskosten für die Auszahlungen zu beiden Zeitpunkten konstant zu halten. Zudem führt der geringe zeitliche Verzug der zweiten Auszahlungsoption dazu, dass das wahrgenommene Risiko der späteren Auszahlung nicht unkontrolliert ansteigt (Laury et al. 2012). Beispielsweise könnten die Teilnehmer erwarten, dass eine Zahlung in einem Jahr ab Experimentdurchführung möglicherweise nicht ausbezahlt würde, da die Experimentdurchführenden die Universität bereits verlassen haben. Die jeweiligen Zeitpunkte der Gewinnauszahlung in drei und zwölf Wochen werden den Teilnehmern visuell durch Kalenderblätter verdeutlicht.

Tabelle 1: Aufbau der CW-task zur Messung der Zeitpräferenz nach Coller/Williams (1999)

\begin{tabular}{|c|c|c|c|c|c|c|}
\hline $\begin{array}{l}\text { Entscheidungs- } \\
\text { situation }\end{array}$ & $\begin{array}{c}\text { Geldbetrag } \\
\text { A } \\
\text { in } 3 \\
\text { Wochen }\end{array}$ & $\begin{array}{l}\text { Bitte wählen } \\
\text { Sie jeweils } \\
\text { Geldbetrag } \\
\text { A oder B }\end{array}$ & $\begin{array}{c}\text { Geldbetrag } \\
\text { B } \\
\text { in } 12 \\
\text { Wochen }\end{array}$ & $\begin{array}{l}\text { Nominaler } \\
\text { Jahreszins }^{\mathrm{a})}\end{array}$ & $\begin{array}{c}\text { Effektiver } \\
\text { Jahreszins }{ }^{\text {a), b) }}\end{array}$ & $\begin{array}{c}\text { Implizierte } \\
\text { Diskontrate bei } \\
\text { einem Wechsel in } \\
\text { der jeweiligen Zeile }{ }^{\mathrm{c})}\end{array}$ \\
\hline 1 & $100,00 €$ & $\mathrm{~A} \circ \circ \mathrm{B}$ & $100,00 €$ & $0,00 \%$ & $0,00 \%$ & $\delta \leq 0,00 \%$ \\
\hline 2 & $100,00 €$ & $\mathrm{~A} \circ \circ \mathrm{B}$ & $100,17 €$ & $1,00 \%$ & $1,01 \%$ & $0,00 \% \leq \delta \leq 1,01 \%$ \\
\hline 3 & $100,00 €$ & $A \circ \circ B$ & $100,35 €$ & $2,00 \%$ & $2,02 \%$ & $1,01 \% \leq \delta \leq 2,02 \%$ \\
\hline$\cdots$ & $\ldots$ & $\ldots$ & $\ldots$ & $\ldots$ & $\ldots$ & $\cdots$ \\
\hline 19 & $100,00 €$ & $A \circ \circ B$ & $118,81 €$ & $100,00 \%$ & $171,46 \%$ & $111,54 \% \leq \delta \leq 171,46 \%$ \\
\hline 20 & $100,00 €$ & $A \circ \circ B$ & $129,48 €$ & $150,00 \%$ & $346,79 \%$ & $171,46 \% \leq \delta \leq 346,79 \%$ \\
\hline
\end{tabular}

a) Die nominalen und effektiven Jahreszinssätze werden nur einer Teilgruppe angezeigt.

b) Die effektiven Jahreszinssätze von $0,00 \%$ bis 346,79\% ergeben sich durch die Berechnung der Tageszinssätze für die 63 Tage zwischen den beiden Auszahlungsterminen hochgerechnet auf ein Jahr.

c) Die implizierten Diskontraten werden den Teilnehmern nicht angegeben.

Quelle: eigene Darstellung nach Laury et al. (2012)

Um die Sensitivität der Methode zur Zeitpräferenzmessung bezüglich der Höhe der angebotenen Geldbeträge zu untersuchen, wird die Methode nicht nur mit Geldbeträgen beginnend bei $100 €(100 €$ Treatment), sondern auch mit dreifachen Geldbeträgen (300€ bei 
Geldbetrag A; $300 €$ bis $388,45 €$ bei Geldbetrag B), aber identischen Zinssätzen, angewendet (300€ Treatment).

\subsubsection{Aufbau der HL-task zur Messung der Risikoeinstellung}

Zur Ermittlung der Risikoeinstellung der Experimentteilnehmer werden diese gebeten, in 20 Entscheidungssituationen jeweils zwischen zwei Lotterien auszuwählen (vgl. Tabelle 2). Mit bestimmten Wahrscheinlichkeiten können in Lotterie A 180,00€ oder 144,00€ gewonnen werden. In Lotterie B können die Teilnehmer 346,50 € oder 9,00 € erhalten. Die Höhe der Geldbeträge in den Lotterien ist so gewählt, dass die Erwartungswerte der Lotterien im Durchschnitt zwischen dem Niveau der Auszahlungen der CW-task mit Geldbeträgen ausgehend von $100 €$ und $300 €$ liegen. Die Gewinnwahrscheinlichkeiten beider Geldbeträge werden über die 20 Entscheidungssituationen systematisch variiert. Die Chance, den jeweils höheren Betrag von 180,00 € bzw. 346,50€ zu erhalten, beträgt in der ersten Entscheidungssituation 5\% und steigt in 5\%-Schritten auf 100\% in Entscheidungssituation 20. Die Wahrscheinlichkeit für den Gewinn des jeweils niedrigeren Betrags in beiden Lotterien entspricht somit $95 \%$ in Entscheidungssituation 1 und sinkt in jeder folgenden Entscheidungssituation um je 5\%. Lotterie B ist im Vergleich zu Lotterie A riskanter, da eine grössere Spannweite der möglichen Ergebnisse besteht.

Tabelle 2: Aufbau der HL-task zur Messung der Risikoeinstellung nach Holt/Laury (2002)

\begin{tabular}{|c|c|c|c|c|c|c|c|}
\hline \multirow{2}{*}{$\begin{array}{l}\text { Ent- } \\
\text { schei- } \\
\text { dungssi- } \\
\text { tuation }\end{array}$} & \multicolumn{2}{|c|}{$\begin{array}{l}\text { Lotterie A } \\
\text { Wahrscheinlichkeit } \\
\text { für den Gewinn von }\end{array}$} & \multirow{2}{*}{$\begin{array}{c}\text { Bitte } \\
\text { wählen Sie } \\
\text { jeweils } \\
\text { Lotterie } \\
\text { A oder B }\end{array}$} & \multicolumn{2}{|c|}{$\begin{array}{l}\text { Lotterie B } \\
\text { Wahrscheinlichkeit } \\
\text { für den Gewinn von }\end{array}$} & \multirow[t]{2}{*}{$\begin{array}{l}\text { Differenz der Er- } \\
\text { wartungswerte }^{\text {a) }}\end{array}$} & \multirow[t]{2}{*}{$\begin{array}{l}\text { Relativer Risikoaver- } \\
\text { sionskoeffizient }^{a} \text { ), b) }\end{array}$} \\
\hline & $180,00 €$ & $144,00 €$ & & $346,50 €$ & $9,00 €$ & & \\
\hline 1 & $5 \%$ & $95 \%$ & $A \circ \circ$ & $5 \%$ & $95 \%$ & $119,93 €$ & $r \leq-2,48$ \\
\hline 2 & $10 \%$ & $90 \%$ & $\mathrm{~A} \circ \circ \mathrm{B}$ & $10 \%$ & $90 \%$ & $104,85 €$ & $-2,48 \leq r \leq-1,71$ \\
\hline 3 & $15 \%$ & $85 \%$ & $A \circ \circ B$ & $15 \%$ & $85 \%$ & $89,78 €$ & $-1,71 \leq r \leq-1,27$ \\
\hline$\cdots$ & $\ldots$ & $\ldots$ & $\ldots$ & $\ldots$ & $\ldots$ & $\ldots$ & $\ldots$ \\
\hline 19 & $95 \%$ & $5 \%$ & $\mathrm{~A} \circ \mathrm{B}$ & $95 \%$ & $5 \%$ & $-151,43 €$ & $1,37 \leq r \leq 1,68$ \\
\hline 20 & $100 \%$ & $0 \%$ & $\mathrm{~A} \circ \mathrm{B}$ & $100 \%$ & $0 \%$ & $-166,50 €$ & $1,68 \leq r \leq 2,25$ \\
\hline
\end{tabular}

a) Die Differenz der Erwartungswerte und der relative Risikoaversionskoeffizient werden den Teilnehmern nicht angezeigt.

b) Bei Annahme einer Potenzrisikonutzenfunktin der Form $u(x)=x^{(1-r)} /(1-r)$.

Quelle: eigene Darstellung nach Laury et al. (2012)

Durch die Beobachtung der Entscheidungssituation, in welcher der jeweilige Teilnehmer von der sichereren Lotterie A zur unsichereren Lotterie B wechselt, kann auf die Risikoeinstellung der Experimentteilnehmer geschlossen werden. Die Erwartungswerte von Lotterie A sind bis Entscheidungssituation 8 höher, ab Entscheidungssituation 9 übersteigen die Erwartungswerte von Lotterie B die von Lotterie A. Risikoneutrale Teilnehmer wählen demnach bis Entscheidungssituation 8 Lotterie A und danach Lotterie B. Ein früherer Wechsel von Lotterie A zu B deutet auf einen risikosuchenden, und ein späterer Wechsel, auf einen risikoaversen Teilnehmer hin. Teilnehmer, die in allen Entscheidungssituationen Option A wählen, ziehen in der letzten Entscheidungssituation den sicheren Gewinn von 180,00€ 
dem sicheren Erhalt von 346,50€ vor, sodass davon ausgegangen werden kann, dass diese Teilnehmer die Aufgabenstellung nicht korrekt verstanden haben.

\subsubsection{Aufbau der L-task zur Messung der Zeitpräferenz}

In der L-task haben die Teilnehmer die Möglichkeit, in 20 Entscheidungssituationen jeweils zwischen zwei Lotterien mit einer potentiellen Auszahlung von $0 €$ oder $100 € \mathrm{zu}$ wählen (vgl. Tabelle 3). In Lotterie A beträgt die Wahrscheinlichkeit für den Gewinn von $100 €$ in allen 20 Entscheidungssituationen 50,0\%. Bei Lotterie B beträgt die Wahrscheinlichkeit $100 € \mathrm{zu}$ gewinnen in Entscheidungssituation 1 50,0\%. Bis Entscheidungssituation 20 steigt die Wahrscheinlichkeit auf 64,7\% an. Die Auszahlung der Geldprämie erfolgt bei Lotterie A - wie in der CW-task - in drei Wochen. Den möglichen Gewinn aus Lotterie B erhalten die Teilnehmer in zwölf Wochen ab Experimentdurchführung. Die Zeitpunkte der Auszahlung werden auf Kalenderblättern visualisiert. Mit dem Wechsel eines Teilnehmers von der Wahl der Lotterie A zur Wahl der Lotterie B wird implizit die individuelle Diskontrate zum Ausdruck gebracht.

Tabelle 3: Aufbau der L-task zur Messung der Zeitpräferenz nach Laury et al. (2012)

\begin{tabular}{|c|c|c|c|c|c|c|}
\hline $\begin{array}{l}\text { Ent- } \\
\text { schei- } \\
\text { dungs- } \\
\text { situa- } \\
\text { tion }\end{array}$ & $\begin{array}{c}\text { Lotterie A } \\
\text { Wahrscheinlichkeit } \\
\text { für den } \\
\text { Gewinn } \\
\text { von } 100 € \\
\text { in } 3 \text { Wochen }\end{array}$ & $\begin{array}{c}\text { Bitte } \\
\text { wählen Sie } \\
\text { jeweils } \\
\text { Lotterie } \\
\text { A oder B }\end{array}$ & $\begin{array}{c}\text { Lotterie B } \\
\text { Wahrscheinlichkeit } \\
\text { für den } \\
\text { Gewinn } \\
\text { von } 100 € \\
\text { in } 12 \text { Wochen }\end{array}$ & $\begin{array}{l}\text { Nomi- } \\
\text { naler } \\
\text { Jahres- } \\
\text { zins }^{\text {a) }}\end{array}$ & $\begin{array}{l}\text { Effek- } \\
\text { tiver } \\
\text { Jahres- } \\
\text { zins }{ }^{\text {a), b) }}\end{array}$ & $\begin{array}{c}\text { Implizierte } \\
\text { Diskontrate } \\
\text { bei einem Wechsel } \\
\text { in dieser Zeile }\end{array}$ \\
\hline 1 & $50,0 \%$ & $\mathrm{~A} \circ \circ \mathrm{B}$ & $50,0 \%$ & $0,00 \%$ & $0,00 \%$ & $\delta \leq 0,00 \%$ \\
\hline 2 & $50,0 \%$ & $A \circ \circ B$ & $50,1 \%$ & $1,00 \%$ & $1,01 \%$ & $0,00 \% \leq \delta \leq 1,01 \%$ \\
\hline 3 & $50,0 \%$ & $A \circ \circ B$ & $50,2 \%$ & $2,00 \%$ & $2,02 \%$ & $1,01 \% \leq \delta \leq 2,02 \%$ \\
\hline$\cdots$ & $\cdots$ & $\ldots$ & $\cdots$ & $\ldots$ & $\ldots$ & $\ldots$ \\
\hline 19 & $50,0 \%$ & $A \circ \circ \mathrm{B}$ & $59,4 \%$ & $100,00 \%$ & $171,46 \%$ & $111,54 \% \leq \delta \leq 171,46 \%$ \\
\hline 20 & $50,0 \%$ & $\mathrm{~A} \circ \circ \mathrm{B}$ & $64,7 \%$ & $150,00 \%$ & $346,79 \%$ & $171,46 \% \leq \delta \leq 346,79 \%$ \\
\hline
\end{tabular}

a) Die nominalen und effektiven Jahreszinssätze sowie die implizierten Diskontraten werden den Teilnehmern nicht angezeigt.

b) Die effektiven Jahreszinssätze von 0,00\% bis 346,79\% ergeben sich durch die Berechnung der Tageszinssätze für die 63 Tage zwischen den beiden Auszahlungsterminen hochgerechnet auf ein Jahr.

Quelle: eigene Darstellung nach Laury et al. (2012)

Um die Sensitivität der Methode zur Messung der Zeitpräferenz bezüglich der Höhe des Geldbetrages zu untersuchen, werden die Lotterien mit dem dreifachen Geldbetrag, also $300 €$, aber identischen Wahrscheinlichkeiten und Zinssätzen durchgeführt.

\subsection{Durchführung des Experiments}

Das Experiment wurde im Januar und Februar 2014 online durchgeführt. Über agrarnahe Verbände, Vereine und Fachzeitschriften wurden Landwirte zur Teilnahme am Experiment eingeladen. Das Experiment wurde von 154 Landwirten vollständig bearbeitet. Da individuelle Präferenzen der Teilnehmer durch deren intuitives Entscheidungsverhalten untersucht werden sollen, wurde zu Beginn des Experiments darauf hingewiesen, dass bei den 
Entscheidungen kein „richtig“ oder „falsch“ existiert. Die Bearbeitungszeit des Experiments betrug durchschnittlich 25 Minuten.

Um den Teilnehmern nicht zu viele Wahlentscheidungen der gleichen Struktur vorzulegen und damit die Bearbeitungszeit des Experiments nicht zu stark auszudehnen, werden zwei Teilgruppen gebildet. Welche der Teilexperimente des Lotterie- und Auswahlteils der jeweiligen Teilnehmergruppe vorgelegt wurden, zeigt Tabelle 4.

Tabelle 4: Schematischer Aufbau des Lotterie- und Auswahlteils des Experiments

\begin{tabular}{|c|c|c|c|c|c|c|c|}
\hline & \multicolumn{4}{|c|}{$\begin{array}{c}\text { CW-task }{ }^{\mathrm{a})} \\
(\text { Teil 1der CW/HL-task) } \\
\text { mit Zinsangabe ohne Zinsangabe }\end{array}$} & \multirow[t]{2}{*}{$\begin{array}{c}\text { HL-task } \\
\text { (Teil 2 der } \\
\text { CW/HL-task) }\end{array}$} & \multicolumn{2}{|c|}{$\begin{array}{l}\text { L-task }^{\text {a) }} \\
\text { ohne Zinsangabe }\end{array}$} \\
\hline & $\begin{array}{l}\text { Basis } \\
100 €\end{array}$ & $\begin{array}{l}\text { Basis } \\
300 €\end{array}$ & $\begin{array}{l}\text { Basis } \\
100 €\end{array}$ & $\begin{array}{l}\text { Basis } \\
300 €\end{array}$ & & $\begin{array}{l}\text { Basis } \\
100 €\end{array}$ & $\begin{array}{l}\text { Basis } \\
300 €\end{array}$ \\
\hline $\begin{array}{l}\text { Teilnehmer- } \\
\text { gruppe } 1\end{array}$ & $\mathrm{x}$ & $\mathrm{x}$ & & & $\mathrm{x}$ & $\mathrm{X}$ & $\mathrm{x}$ \\
\hline $\begin{array}{l}\text { Teilnehmer- } \\
\text { gruppe } 2\end{array}$ & & & $\mathrm{x}$ & $\mathrm{x}$ & $\mathrm{x}$ & $\mathrm{x}$ & $\mathrm{x}$ \\
\hline
\end{tabular}

a) Die Abfolge der Teilexperimente ist randomisiert: Vor und nach der Risikoeinstellungsmessung wird jeweils ein Mal die CW-task und ein Mal die L-task mit zufälligen Geldbeträgen durchgeführt.

Quelle: eigene Darstellung

Die Anordnung der CW-task und der L-task sowie die Anordnung der Treatments (100€ und $300 €)$ vor oder hinter der HL-task ist randomisiert. Die Messung der Risikoeinstellung mit der HL-task wird, der Vorgehensweise von Laury et al. (2012) folgend, immer an dritter Stelle des Experiments durchgeführt. Das heisst, vor der Messung der Risikoeinstellung werden in zufälliger Abfolge und mit einem zufälligen Treatment die CW-task und die L-task durchgeführt. Die beiden Methoden werden in der Abfolge gleichbleibend im alternativen Treatment im Anschluss an die HL-task durchgeführt. Damit ergeben sich vier mögliche Abfolgen der Teilaufgaben im Experiment. Diese Randomisierung dient zur Steigerung der internen Validität und Reliabilität der Ergebnisse (Harrison et al. 2009).

Um die Motivation der Landwirte zur Teilnahme zu erhöhen, aber auch um reale Entscheidungssituationen zu schaffen, werden alle Teilexperimente an monetäre Anreize gekoppelt. Für die Teilnehmer besteht mit einer Wahrscheinlichkeit von 10\% die Möglichkeit, eine Geldprämie zu gewinnen. Für jeden der als Gewinner ausgewählten Teilnehmer wird eine zufällig ausgewählte Entscheidung innerhalb der durchgeführten Lotterien bzw. Wahlentscheidungen auszahlungsrelevant. Bei allen Lotterien und Wahlentscheidungen legen die Angaben der Teilnehmer die Höhe und den Zeitpunkt des möglichen Gewinns fest.

\subsection{Bestimmung der Diskontraten für die CW/HL-task}

Um die individuelle Diskontrate der CW/HL-task zu ermitteln, müssen die individuellen Diskontraten aus der CW-task mit der festgestellten individuellen Risikoeinstellung aus der HL-task korrigiert werden.

Bei der Bestimmung der Diskontraten wird nach Andersen et al. (2008) und auf der Grundlage der zeitlichen Verzögerung der ersten möglichen Auszahlung exponentielles Diskontieren angenommen. Ausserdem zeigen Andersen et al. (2014) mit experimentellen 
Daten einer repräsentativen Stichprobe der dänischen Bevölkerung, dass die ermittelten Diskontraten bei Annahme von exponentiellem oder hyperbolischem Diskontieren keine signifikanten Unterschiede aufweisen. Durch Gleichsetzen der Barwerte beider Optionen A und B beim Wechsel eines Teilnehmers von Option A zu Option B in der Zeitpräferenzmessung mit CW-task ergibt sich:

$$
\mathrm{U}\left(\omega+\mathrm{M}_{\mathrm{A}}\right)+\left(\frac{1}{1+\delta}\right)^{\tau} \mathrm{U}(\omega)=\mathrm{U}(\omega)+\left(\frac{1}{1+\delta}\right)^{\tau} \mathrm{U}\left(\omega+\mathrm{M}_{\mathrm{B}}\right)
$$

Dabei stellt $\mathrm{U}(\bullet)$ den Nutzen dar. Der Grundkonsum $\omega$ und der, über das Treatment definierte Geldbetrag $M_{A}(100 €$ bzw. $300 €)$, sind feste Parameter. $M_{B}$ stellt den Geldbetrag dar, bei dem der Teilnehmer zwischen den Geldbeträgen $\mathrm{M}_{\mathrm{A}}$ und $\mathrm{M}_{\mathrm{B}}$ indifferent ist. ${ }^{1}$ Der Indifferenzbetrag eines Teilnehmers ist der Mittelwert des Geldbetrages B bei der letzten Wahl des Geldbetrags A des Teilnehmers und seines ersten gewählten Geldbetrags B. Wählt ein Teilnehmer z. B. in der dritten Entscheidungssituation der CW-task im $100 €$ Treatment das erste Mal B, so beträgt $M_{B} 100,26 €((100,17 €+100,35 €) / 2)$. Die Diskontrate $\delta$ ist Teil des Diskontierungsfaktors, mit welchem der zukünftige Nutzen der Geldbeträge über die Zeitspanne $\tau$ abgezinst wird. In unserem Fall erstreckt sich die Zeitspanne $\tau$ über 63 Tage und beträgt somit 63/365. Die Zeitspanne $\tau$ stellt also die Differenz zwischen dem letzten und ersten Auszahlungszeitpunkt in zwölf bzw. drei Wochen dar.

Es wird ausserdem von einer Potenz-Risikonutzenfunktion der Teilnehmer ausgegangen, die einen konstanten relativen Risikoaversionskoeffizienten (constant relative risk aversion; CRRA) r impliziert (vgl. z.B. Holt/Laury 2002; Andersen et al. 2008; Eckel/Grossman 2008):

$$
\mathrm{U}(\mathrm{M})=\frac{(\omega+\mathrm{M})^{(1-\mathrm{r})}}{(1-\mathrm{r})}
$$

Der CRRA-Wert lässt sich aus der Messung der Risikoeinstellung mit der HL-task ablesen. Es wird immer der Mittelwert (nach Harrison et al. 2007) der mit dem Wechsel assoziierten CRRA Spanne verwendet (vgl. Tabelle 2, Spalte 6). Für den Fall, dass ein Teilnehmer bereits in der ersten Entscheidungssituation Lotterie B wählt (was für einen Teilnehmer zutrifft), wird ein CRRA Wert von -2,48 verwendet. Durch Einsetzen von (2) in (1) geht die Risikoeinstellung der Teilnehmer in die Zeitpräferenzmessung ein. Daraus folgend können die Diskontraten $\delta$ der CW/HL-task nach dem Vorbild von Andersen et al. (2008) bestimmt werden:

$$
\delta=\left(\frac{\left(\omega^{(1-r)}-\left(\omega+\frac{M_{B}}{\lambda}\right)^{(1-r)}\right)}{\left(\omega^{(1-r)}-\left(\omega+\frac{M_{A}}{\lambda}\right)^{(1-r)}\right)}\right)^{\frac{1}{\tau}}-1
$$

1 Es wird Andersen et al. (2008) folgend angenommen, dass die Integration des Geldgewinns in den Konsum innerhalb eines Tages stattfindet. 


\section{Deskriptive Statistik und Ansatz zur Datenauswertung}

Im folgenden Abschnitt werden, im Rahmen der deskriptiven Statistik, die soziodemographischen, sozioökonomischen und betrieblichen Charakteristika der Teilnehmergruppe dargestellt. Anschliessend wird der Ansatz zur Datenanalyse begründet und der verwendete Regressionsansatz erklärt.

\subsection{Deskriptive Statistik}

30 der 154 Teilnehmer wechseln in einem der Teilexperimente nach dem Wechsel von Option A zu Option B wieder zurück zu Option A. Sie weisen also ein inkonsistentes Entscheidungsverhalten auf (Thaler 1981; Pender 1996; Laury et al. 2012). Dieses inkonsistente Entscheidungsverhalten gilt auch für drei weitere Teilnehmer, die in der HL-task in der 20. Entscheidungssituation Lotterie A wählen und somit den sicheren Erhalt von 180,00 € dem sicheren Gewinn von 346,50€ vorziehen. Der Anteil inkonsistenter Teilnehmer dieser Untersuchung unterschreitet in jedem Teilexperiment den Anteil der inkonsistenten Teilnehmer der mit 103 Studierenden durchgeführten Untersuchung von Laury et al. (2012). Für die CW-task finden Laury et al. (2012) bei 21,36\% der Probanden inkonsistente Entscheidungen, wohingegen in dieser Untersuchung nur 10,39\% ein inkonsistentes Entscheidungsverhalten zeigen. In der HL-task sind bei Laury et al. (2012) 22,33\% der Teilnehmer bei ihren Entscheidungen inkonsistent, in unserer Untersuchung lediglich $16,23 \%$. Auch bei der L-task sind unsere Teilnehmer prozentual weniger inkonsistent $(11,69 \%)$ als die Teilnehmer in der Studie von Laury et al. (2012) mit 25,24\%. Insbesondere bei der HL-task kommt es zu inkonsistentem Verhalten, was bereits in anderen Untersuchungen festgestellt wurde (Jacobson/Petrie 2009; Herberich/List 2012).

Die soziodemografischen Charakteristika sowie die Strukturen der bewirtschafteten Betriebe der 121 Landwirte, die in allen Teilexperimenten konsistentes Entscheidungsverhalten gezeigt haben, und der 33 Landwirte, die sich in mindestens einem der Teilexperimente nicht konsistent verhalten haben, sind in Tabelle 5 dargestellt. Tabelle 5 können ausserdem die Ergebnisse der nichtparametrischen Mann-Whitney-U-Tests für unabhängige Stichproben entnommen werden, mit welchen untersucht wird, ob sich beide Gruppen in ihren Eigenschaften signifikant unterscheiden.

Tabelle 5: Deskriptive Statistik der Teilnehmer mit im Experiment konsistentem und inkonsistentem Entscheidungsverhalten

\begin{tabular}{|c|c|c|c|c|c|}
\hline \multirow[t]{2}{*}{ Variable } & \multicolumn{2}{|c|}{$\begin{array}{c}\text { Teilnehmer mit } \\
\text { konsistentem Entschei- } \\
\text { dungsverhalten ( } \mathrm{N}=121)\end{array}$} & \multicolumn{2}{|c|}{$\begin{array}{l}\text { Teilnehmer mit inkonsis- } \\
\text { tentem Entscheidungsver- } \\
\text { halten }(\mathrm{N}=33)\end{array}$} & \multirow{2}{*}{ p-Wert ${ }^{\text {a) }}$} \\
\hline & Mittelwert & $\begin{array}{l}\text { Standard- } \\
\text { abweichung }\end{array}$ & Mittelwert & $\begin{array}{l}\text { Standard- } \\
\text { abweichung }\end{array}$ & \\
\hline Alter der Teilnehmer (in Jahren) & 34,40 & 12,76 & 45,03 & 15,31 & $0,001 * * *$ \\
\hline Anteil weiblicher Teilnehmer (in \%) & \multicolumn{2}{|c|}{10,74} & \multicolumn{2}{|c|}{6,10} & \\
\hline Bildungsjahre (in Jahren) ${ }^{\text {b) }}$ & 14,46 & 3,16 & 12,72 & 3,24 & $0,007 * * *$ \\
\hline $\begin{array}{l}\text { Anteil Teilnehmer mit landwirtschaftlichem } \\
\text { Abschluss }^{c} \text { (in \%) }\end{array}$ & \multicolumn{2}{|c|}{90,91} & \multicolumn{2}{|c|}{96,97} & \\
\hline HL-Wert ${ }^{\text {d) }}$ & 10,98 & 4,03 & / & l & \\
\hline
\end{tabular}




\begin{tabular}{|c|c|c|c|c|c|}
\hline \multirow[t]{2}{*}{ Variable } & \multicolumn{2}{|c|}{$\begin{array}{c}\text { Teilnehmer mit } \\
\text { konsistentem Entschei- } \\
\text { dungsverhalten }(\mathrm{N}=121)\end{array}$} & \multicolumn{2}{|c|}{$\begin{array}{l}\text { Teilnehmer mit inkonsis- } \\
\text { tentem Entscheidungsver- } \\
\text { halten }(\mathrm{N}=33)\end{array}$} & \multirow[t]{2}{*}{ p-Wert ${ }^{\text {a) }}$} \\
\hline & Mittelwert & $\begin{array}{c}\text { Standard- } \\
\text { abweichung }\end{array}$ & Mittelwer & $\begin{array}{l}\text { Standard- } \\
\text { abweichung }\end{array}$ & \\
\hline $\begin{array}{l}\text { Selbsteinschätzung der Vermögenssituation } \\
\text { im Vergleich zu anderen } \\
\text { Betriebsleitern e) }\end{array}$ & 5,56 & 1,53 & 5,09 & 1,28 & 0,222 \\
\hline Bewirtschaftete Fläche (in ha) & 198,01 & 421,95 & 123,41 & 132,59 & 0,900 \\
\hline Anteil Haupterwerbsbetriebe (in \%) & \multicolumn{2}{|c|}{80,17} & \multicolumn{2}{|c|}{72,73} & \\
\hline $\begin{array}{l}\text { Anteil ökologisch wirtschaftender } \\
\text { Betriebe (in \%) }\end{array}$ & \multicolumn{2}{|c|}{6,61} & \multicolumn{2}{|c|}{3,03} & \\
\hline \multicolumn{6}{|l|}{ Betriebsausrichtungen } \\
\hline $\begin{array}{l}\text { Anteil Betriebe mit dem Betriebszweig } \\
\text { Ackerbau (in \%) }\end{array}$ & \multicolumn{2}{|c|}{74,38} & \multicolumn{2}{|c|}{75,76} & \\
\hline $\begin{array}{l}\text { Anteil Betriebe mit dem Betriebszweig } \\
\text { Veredelung (in \%) }\end{array}$ & \multicolumn{2}{|c|}{34,71} & \multicolumn{2}{|c|}{36,36} & \\
\hline $\begin{array}{l}\text { Anteil Betriebe mit dem Betriebszweig } \\
\text { Milchvieh (in \%) }\end{array}$ & \multicolumn{2}{|c|}{28,93} & \multicolumn{2}{|c|}{24,24} & \\
\hline $\begin{array}{l}\text { Anteil Betriebe mit dem Betriebszweig } \\
\text { Biogas (in \%) }\end{array}$ & \multicolumn{2}{|c|}{14,87} & \multicolumn{2}{|c|}{9,68} & \\
\hline $\begin{array}{l}\text { Anteil Betriebe mit dem Betriebszweig } \\
\text { Obstbau (in \%) }\end{array}$ & \multicolumn{2}{|c|}{12,40} & \multicolumn{2}{|c|}{6,45} & \\
\hline Anteil Betriebe mit Nachfolger (in \%) & \multicolumn{2}{|c|}{75,20} & \multicolumn{2}{|c|}{66,74} & \\
\hline \multicolumn{6}{|c|}{ a) Mann-Whitney-U-Test: $*(* *, * * *)$ bedeutet $\mathrm{p}$-Wert $<0,10(\mathrm{p}$-Wert $<0,05, \mathrm{p}$-Wert $<0,01)$} \\
\hline \multicolumn{6}{|c|}{ b) Ohne Berufsschule; nach dem Umrechnungsschlüssel der OECD (1999) } \\
\hline \multicolumn{6}{|c|}{$\begin{array}{l}\text { c) Eine landwirtschaftliche Ausbildung kann von einer landwirtschaftlichen Lehre bis zu einem Studium } \\
\text { der Agrarwissenschaften reichen. }\end{array}$} \\
\hline \multicolumn{6}{|c|}{ d) $0-7=$ risikosuchend, $8=$ risikoneutral, 9-19 = risikoavers } \\
\hline \multicolumn{6}{|c|}{ e) $0-4=$ unterdurchschnittlich, $5=$ durchschnittlich, 6-10 = überdurchschnittlich } \\
\hline
\end{tabular}

Die Landwirte mit konsistentem Entscheidungsverhalten im Experiment sind mit einem Durchschnittsalter von 34,40 Jahren relativ jung, wobei der jüngste Experimentteilnehmer 18 Jahre alt ist und der älteste 58 Jahre alt ist. Ausserdem können die Landwirte mit einem durchschnittlichen HL-Wert („number of safe choices“) von 10,98 nach Holt/Laury (2002) als leicht risikoavers eingestuft werden. Dieses Ergebnis ist übereinstimmend mit Ergebnissen der Risikoeinstellungsmessung von Landwirten durch Maart-Noelck/Mußhoff (2013) und durch Reynaud/Couture (2012). Die durchschnittliche Flächenausstattung der Betriebe von konsistent entscheidenden Betriebsleitern beträgt 198,01 ha, wobei der kleinste Betrieb 5 ha Sonderkulturen und der grösste 3.200 ha Ackerfläche, sowie 450 ha Grünland, bewirtschaftet.

Die Landwirte mit konsistentem Entscheidungsverhalten sind im Vergleich zu den Landwirten mit inkonsistentem Entscheidungsverhalten signifikant jünger $(\mathrm{p}<0,01$; MannWhitney-U-Test). Ebenso unterscheiden sich beide Gruppen in der Anzahl der mit unserem Fragebogen erfassten Bildungsjahre. Konsistent entscheidende Landwirte haben signifikant ( $\mathrm{p}<0,01$; Mann-Whitney-U-Test) mehr Bildungsjahre absolviert.

Die ermittelten Diskontraten für die verschiedenen Methoden und Treatments der konsistenten Landwirte zeigt Tabelle 6. Inkonsistente Teilnehmer werden bei der Bestimmung der individuellen Diskontrate nicht berücksichtigt, da ihnen keine eindeutigen Werte zuzu- 
ordnen sind (Hirschauer et al. 2014). Wird die Risikoaversion der Teilnehmer berücksichtigt, so sinken die in der CW-task gemessenen Diskontraten. Diese Tendenz ist auch bei den Untersuchungen von Laury et al. (2012) und Andersen et al. (2008) festzustellen und spiegelt die durchschnittlich leicht risikoaverse Einstellung der Teilnehmergruppe wider.

Tabelle 6: Implizierte jährliche Diskontraten nach den verschiedenen Methoden zur Messung der Diskontrate

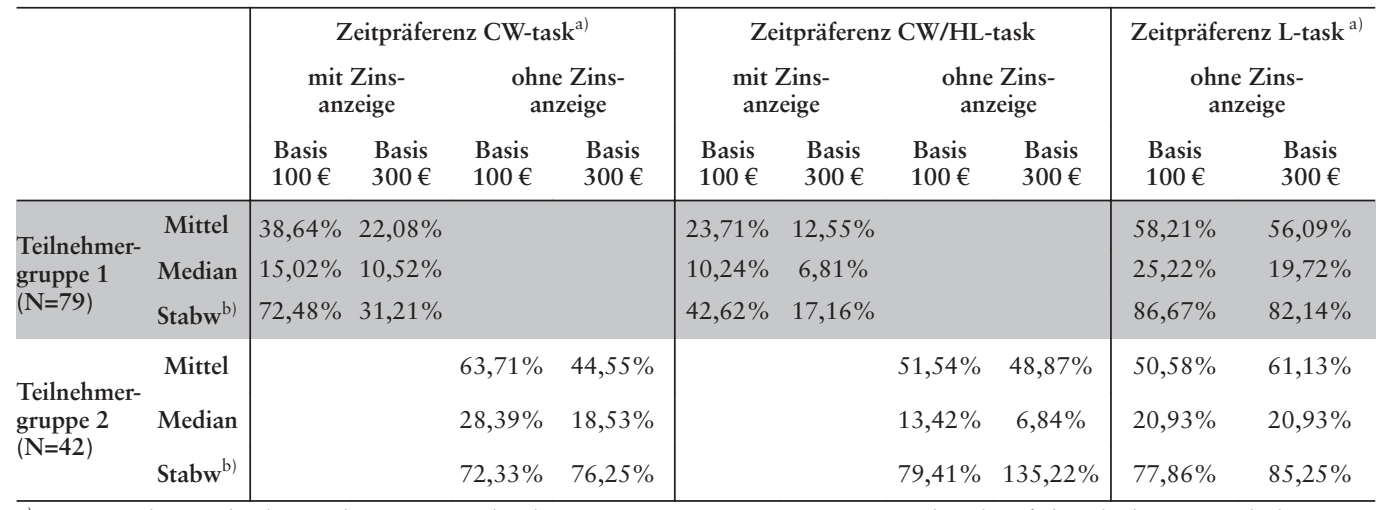

a) Wenn die Teilnehmer keinen Wechsel von Option A zu Option B durchgeführt haben, wird das Entscheidungsverhalten wie ein Wechsel in Entscheidungssituation 21 betrachtet (Coller/Williams 1999) und eine Diskontrate von 346,79\% pro Jahr angenommen.

b) Stabw $=$ Standardabweichung.

Quelle: eigene Berechnung

Aus der deskriptiven Darstellung der Diskontraten wird die Tendenz einer sinkenden Diskontrate bei Verwendung des $300 €$ Treatments für die CW/HL-task ersichtlich. Bei der Ltask ist diese Tendenz nicht zu erkennen. Nachfolgend wird ein kurzer Überblick über Diskontraten in anderen Studien gegeben. Zunächst werden einige Arbeiten angeführt, die mit der festgestellten Diskontrate deutlich unterhalb der hier gefundenen liegen. So ermitteln Beckers et al. (2009) eine Diskontrate von 1,97\% für Wirtschaftlichkeitsuntersuchungen der öffentlichen Verwaltung. Auch Alfen Consult et al. (2014) verwenden eine niedrige Diskontrate von 1,10\% für ihre Wegekostenberechnung. Beide Arbeiten leiten diese Diskontraten aus dem Konzept der sozialen Zeitpräferenzrate nach der Ramsey-Gleichung ab. Link et al. (2009) verwendet eine Diskontrate von 2,50\% für die deutsche Wegekostenberechnung und beziehen diese Diskontrate aus dem Opportunitätskostenansatz, welcher Anleihen der öffentlichen Hand als Bezugsgrösse verwendet. Hausman (1979) weisen auf felddatenbasierte statistisch-ökonometrisch geschätzte Diskontraten um $20 \%$ hin. Harrison et al. (2002) zeigen für die dänische Bevölkerung experimentell eine Diskontrate von $28 \%$. Auch andere experimentelle Arbeiten liegen mit den ermittelten Diskontraten im Bereich der Mediane unserer Diskontraten (6,81\% bis 25,22\%). So ermitteln Andersen et al. (2008) eine Diskontrate von 10,10\%. Laury et al. (2012) zeigen, dass die Diskontrate je nach Methode 12,20\% bzw. 14,10\% beträgt. 


\subsection{Ansatz zur Datenanalyse}

Im Folgenden wird dargestellt, wie unsere in Kapitel 2 aufgestellten Hypothesen mit den experimentell gewonnenen Daten überprüft werden können. Die Wahl der Analysemethode begründet sich aus der Struktur der Daten. Da pro Teilnehmer vier Diskontraten ermittelt werden, die sich aus der Anwendung beider Methoden in der beiden Treatments ergeben, sind die im Experiment gewonnenen Diskontraten nicht als unabhängige Beobachtungen zu werten. Auf Grund dieser Abhängigkeitsstruktur der Beobachtungen wird ein „Generalisiertes Lineares Gemischtes Modell“ (GLMM) der abhängigen Variable $y$ über die Personen $i$ und die Zeitpunkte $j$ geschätzt (Fahrmeir et al. 2013):

$$
\mathrm{y}_{\mathrm{ij}}=\beta_{0}+\beta_{1} \mathrm{x}_{\mathrm{ij} 1}+\ldots+\beta_{\mathrm{k}} \mathrm{x}_{\mathrm{ijk}}+\gamma_{0 \mathrm{i}}+\varepsilon_{\mathrm{ij}}
$$

Dabei bezeichnet $\beta_{0}$ den globalen Achsenabschnitt. $\beta_{1}, \ldots, \beta_{k}$ beschreiben die Steigungsparameter der unabhängigen Variablen $1, \ldots, k$ mit den einzelnen Ausprägungen $x_{i j 1}, \ldots, x_{i j k}$. Der aus dem klassischen linearen Modell bekannte Fehler wird durch $\varepsilon_{i j}$ mit $\varepsilon_{i j}^{i . i . d .} N\left(0, \sigma^{2}\right)$ symbolisiert. Der personenspezifische random intercept $\gamma_{0 i}$ wird als zufällig angenommen, mit $\gamma_{0 i} \stackrel{\text { i.i.d. }}{\sim} N\left(0, \tau_{0}^{2}\right)$ und der Index $j$ beschreibt alle vier von Teilnehmer $i$ erfassten Diskontfaktoren. Die Schätzung eines GLMM ist nicht an die Normalverteilungsannahme der abhängigen Variablen gebunden. Stattdessen wird mittels QQ-Plot eine passende Verteilung identifiziert und ein Modell mit der entsprechend optimalen Verteilung geschätzt.

Um die aufgestellten Hypothesen zu beantworten, werden die experimentell erhobenen Daten in das beschriebene Modell integriert. Zunächst werden die ermittelten Diskontfaktoren $(1 /(1+\delta))$, welche für jeden Teilnehmer i auf vier unterschiedliche Arten erfasst wurden, als abhängige Variable y genutzt. Durch die Verwendung der Diskontfaktoren ist die Interpretation der Koeffizienten spiegelbildlich zu einer Verwendung der Diskontraten; Eine steigende Diskontrate ist gleichbedeutend mit einem sinkenden Diskontfaktor. Um zu überprüfen, ob die Methoden zu identischen Diskontfaktoren führen, und um den Einfluss der Treatments zu untersuchen, werden verschiedene Variablen integriert: Die CW/HLtask mit und ohne angezeigte Zinssätze werden als Dummy-Variablen aufgenommen ('Dummy CW/HL mit Zins'; 'Dummy CW/HL ohne Zins'). Neben diesen Variablen wird eine Dummy-Variable für das $300 €$ Treatment in das Modell aufgenommen ('Dummy $\left.300 €^{\prime}\right)$. Um jede der drei Erfassungsarten in den jeweiligen Treatments prüfen zu können, werden zusätzlich zwei Interaktionen aufgenommen. Eine Interaktion beschreibt den Effekt, dass zwei unabhängige Variablen in Abhängigkeit von der Ausprägung der jeweils anderen auf die y-Variable wirken. Die Interaktionen zeigen den Effekt bei zeitgleicher Anwendung der CW/HL-task mit Zins und des $300 €$ Treatments ('CW/HL mit Zins • $300 €$ '), beziehungsweise bei zeitgleicher Anwendung der CW/HL-task ohne Zins und des $300 €$ Treatments ('CW/HL ohne Zins • $300 €$ '). Als Referenz dienen folglich die in der Ltask (im $100 €$ Treatment) erfassten Diskontfaktoren. Zudem werden folgende soziodemographische und sozioökonomische Charakteristika der Teilnehmer berücksichtigt: Alter, Geschlecht, Bildungsjahre und die Selbsteinschätzung der Vermögenssituation. Ebenso werden betriebsspezifische Angaben, wie die Erwerbsform des Betriebes, das Vorhanden- 
sein eines Nachfolgers, die Wirtschaftsweise und die Grösse des Betriebes (über die Proxy der bewirtschafteten Flächen in ha) aufgenommen.

\section{Hypothesenüberprüfung}

Zur Überprüfung der Hypothesen werden die ermittelten individuellen Diskontfaktoren herangezogen. Die Ergebnisse der Regressionsanalyse zeigt Tabelle 7.

Tabelle 7: Ergebnisse des GLMM zur Erklärung der ermittelten Diskontfaktoren $(\mathrm{N}=484)$

\begin{tabular}{|c|c|c|}
\hline Erklärende Variable & Koeffizient & p-Wert ${ }^{a)}$ \\
\hline Konstante & 0,777 & $<0,010 * * *$ \\
\hline $\begin{array}{l}\text { Dummy } C W / H L \text { mit Zins } \\
\text { (1 = angewendet) }\end{array}$ & 0,110 & $<0,010 * * *$ \\
\hline $\begin{array}{l}\text { Dummy } C W / H L \text { ohne Zins } \\
\text { (1 = angewendet })\end{array}$ & 0,016 & 0,597 \\
\hline $\begin{array}{l}\text { Dummy } 300 € \\
(1=\text { angewendet })\end{array}$ & $-0,007$ & 0,726 \\
\hline $\begin{array}{l}C W / H L \text { mit Zins } \bullet 300 € \\
(1=\text { angewendet })\end{array}$ & 0,051 & $0,091 *$ \\
\hline $\begin{array}{l}C W / H L \text { ohne } \mathrm{Zins} \bullet 300 € \\
(1=\text { angewendet })\end{array}$ & 0,072 & $0,044 * *$ \\
\hline $\begin{array}{l}\text { Alter } \\
\text { (in Jahren) }\end{array}$ & 0,001 & 0,800 \\
\hline $\begin{array}{l}\text { Geschlecht } \\
(1=\text { weiblich })\end{array}$ & 0,010 & 0,844 \\
\hline $\begin{array}{l}\text { Bildungsjahre } \\
\text { (in Jahren) }\end{array}$ & $-0,004$ & 0,394 \\
\hline Selbsteinschätzung der Vermögenssituation ${ }^{\mathrm{b})}$ & 0,001 & 0,964 \\
\hline $\begin{array}{l}\text { Bewirtschaftete Fläche } \\
\text { (in ha) }\end{array}$ & 0,001 & 0,294 \\
\hline $\begin{array}{l}\text { Dummy Erwerbsform } \\
(1=\text { Nebenerwerb) }\end{array}$ & $-0,045$ & $0,085 *$ \\
\hline $\begin{array}{l}\text { Dummy Wirtschaftsweise } \\
\text { (1= ökologisch) }\end{array}$ & $-0,003$ & 0,965 \\
\hline $\begin{array}{l}\text { Dummy Betriebsleiternachfolger } \\
\text { (1= nicht vorhanden) }\end{array}$ & $-0,011$ & 0,759 \\
\hline Random Intercept & & $<0,010 * * *$ \\
\hline Korrigiertes $\mathbf{R}^{2}$ & & 0,481 \\
\hline
\end{tabular}

a) $*(* * * * *)$ bedeutet $\mathrm{p}$-Wert $<0,10(\mathrm{p}$-Wert $<0,05, \mathrm{p}$-Wert $<0,01)$

b) Variablenwerte 0-10, mit der Bedeutung: 0-4 = unterdurchschnittlich, $5=$ durchschnittlich, 6-10 = überdurchschnittlich

Quelle: eigene Berechnung 
Die Robustheit der Schätzergebnisse wurde mittels Veränderung des Regressionsmodells (Schätzung ohne nicht signifikante Variablen) und mittels Wilcoxon-Vorzeichen-RangTests geprüft. Die Ergebnisse bleiben qualitativ bei jeder durchgeführten Modifikation gleich.

\subsection{Test der Hypothesen $1 a$ und $1 b$}

Wie aus den in Tabelle 7 dargestellten Ergebnissen deutlich wird, führt die Anwendung der CW/HL-task mit Zinsangabe zu höchst signifikant $(\mathrm{p}<0,01)$ anderen Diskontfaktoren als die L-task. Die Diskontfaktoren aus der CW/HL-task sind im Vergleich zu den Diskontfaktoren aus der L-task grösser. Dies gilt sowohl für das $100 €$ Treatment als auch für das $300 €$ Treatment. Zu erkennen sind diese Ergebnisse an den Koeffizienten und Signifikanzen der Variablen Dummy CW/HL mit Zins, Dummy $300 €$ und der Interaktion $C W / H L$ mit Zins $\bullet 300 €$.

Hypothese $1 a$, dass die Zeitpräferenzmessung mittels CW/HL-task und L-task bei der Anwendung auf Landwirte zu nicht signifikant verschiedenen Ergebnissen führen, kann daher nicht bekräftigt werden. Unser Ergebnis steht damit im Widerspruch zu dem Ergebnis, das Laury et al. (2012) in ihrer Untersuchung festgestellt haben. Sie finden keine signifikanten Unterschiede zwischen den nach beiden Methoden ermittelten Diskontraten bei Studierenden. So werden mit Geldbeträgen ausgehend von 200 \$ Diskontraten von 14,1\% mit der CW/HL-task und von 12,2\% in der L-task festgestellt. Diese Diskontraten entsprechen etwa den Diskontfaktoren von 0,87 (14,1\%) und 0,89 (12,2\%). Dass beide Methoden zum gleichen Ergebnis führen kann bei Landwirten nicht bestätigt werden, was auf ein unterschiedliches Entscheidungsverhalten zwischen Landwirten und Studierenden schliessen lässt.

Hypothese $1 b$, dass ein Verzicht auf ein explizites Ausweisen der Zinssätze im Experiment in der CW/HL-task zu signifikant unterschiedlichen Ergebnissen verglichen mit der L-task führt, kann nicht eindeutig beantwortet werden. Die Hypothese kann für das $100 €$ Treatment nicht bestätigt werden, was aus der Variable 'CW/HL ohne Zins' hervor geht. Bei der Verwendung des $300 €$ Treatments kann ein signifikanter Unterschied zwischen den Diskontfaktoren aus der CW/HL-task und der L-task festgestellt werden. Der signifikant positive Koeffizient der Interaktion 'Dummy $C W / H L$ ohne Zins • $300 €$ ' lässt diese Rückschlüsse zu. Hypothese $1 b$, dass der Verzicht auf das explizite Ausweisen der Zinssätze bei der CW/HL-task zu signifikant unterschiedlichen Ergebnissen der beiden Methoden führt, kann bei Durchführung der Zeitpräferenzmessungen im $100 €$ Treatment nicht unterstützt werden. Die Ergebnisse der Durchführung im $300 €$ Treatment unterstützten dagegen Hypothese 1b. Folglich kann die Ursache für den in Hypothese 1a festgestellten Unterschied zwischen den Methoden nicht eindeutig auf das explizite Ausweisen des Zinssatzes bei der Erfassung zurückgeführt werden.

\subsection{Test der Hypothese 2}

Die Anwendung des $300 €$ Treatments, anstelle der Durchführung der Zeitpräferenzmessungen im $100 €$ Treatment, zeigt nicht bei beiden Methoden den gleichen Effekt. In der CW/HL-task sinkt die implizierte Diskontrate im Durchschnitt von 23,71\% auf 12,55\% pro Jahr, wenn die Zeitpräferenzmessungen im $300 €$ Treatment anstatt im $100 €$ Treatment durchgeführt wird und den Teilnehmern die Zinssätze angegeben werden (vgl. Tabel- 
le 6). Diese Veränderung ist signifikant, was sich aus dem p-Wert der Interaktionsvariable 'CW/HL mit Zins • $300 €$ ' in Tabelle 7 ergibt. Auch wenn bei der Experimentdurchführung auf das explizite Ausweisen der Zinssätze in der CW/HL-task verzichtet wird, kommt es im $100 €$ und $300 €$ Treatment zu signifikant unterschiedlichen Ergebnissen. Diesen Rückschluss lässt der signifikante Koeffizient der Interaktionsvariable ' $\mathrm{CW} / \mathrm{HL}$ ohne Zins • $300 €$ ’ zu. Die Diskontfaktoren aus der L-task unterscheiden sich dagegen durch eine Veränderung der Geldbeträge nicht, was der nicht signifikante Koeffizient der Variable 'Dummy 300 €' zeigt. Daher kann Hypothese 2, dass sich die Ergebnisse aus den Zeitpräferenzexperimenten bei beiden Methoden mit veränderten Geldbeträgen signifikant unterscheiden, für die L-task nicht unterstützt werden, für die CW/HL-task allerdings schon.

Der in der CW/HL-task festgestellte Unterschied der Diskontfaktoren bei einer Veränderung der Geldbeträge steht in Einklang mit den Ergebnissen des Experiments von Pender (1996) mit südindischen Landwirten und Landarbeitern. Der Median der ermittelten Diskontraten liegt laut Pender (1996) bei 50\%, bei höheren im Experiment eingesetzten Werten war die Diskontrate niedriger. Die Ergebnisse dieses Experiments lassen sich schwer mit unseren Ergebnissen vergleichen, da Pender (1996) statt Geldprämien Reis als Gut einsetzt und deutlich längere Zeithorizonte wählt. Bocquého et al. (2013) finden einen gegenteiligen Effekt: Die Diskontraten französischer Landwirte erhöhten sich mit steigenden Geldbeträgen leicht. Auch in diesem Fall ist ein Vergleich schwierig, da Bocquébo et al. (2013) Diskontraten unter der Annahme eines linearer Nutzenverlaufs (Risikoneutralität) bestimmen. Die Ergebnisse von Bocquého et al. (2013) widersprechen dem nach Frederick et al. (2002) in vielen Arbeiten zu beobachtenden „magnitude effect“, der besagt, dass mit steigenden Gelbeträgen die Diskontrate sinkt.

\subsection{Weitere Ergebnisse}

Neben den bereits erläuterten Ergebnissen sind Tabelle 7 weitere Ergebnisse zu entnehmen. So ist bis auf die Variable 'Erwerbsform' keine weitere Variable signifikant. Die Erwerbsform hingegen hat einen signifikant negativen Einfluss auf den Diskontfaktor, was bedeutet, dass Betriebsleiter, die ihren Betrieb im Nebenerwerb bewirtschaften, einen signifikant kleineren Diskontfaktor über alle Methoden aufweisen. Ein kleinerer Diskontfaktor könnte somit durch die Erwirtschaftung zusätzlichen Einkommens ausserhalb der Landwirtschaft bedingt werden. Sowohl Tanaka et al. (2010) als auch Bocquého et al. (2013) stellen einen signifikant negativen Einfluss der Bildung auf die Diskontraten fest, sodass mit höherer Bildung die Diskontrate sinkt. Nach Pender (1996) beeinflusst die Bildung die Diskontrate dagegen nicht signifikant. Zum gleichen Ergebnis kommt Pender (1996) auch für den Einfluss des Geschlechts. Sowohl bei der Bildung als auch beim Geschlecht können die Ergebnisse von Pender (1996) eher unterstützt werden. Tanaka et al. (2010) und Bocquého et al. (2013) zeigen einen signifikant negativen Einfluss des Alters auf die Diskontrate. Dieser kann von Pender (1996) und auch durch unsere Ergebnisse nicht bestätigt werden. Eine signifikant negative Abhängigkeit der Diskontrate von der Vermögenssituation wird von Pender (1996) und Tanaka et al. (2010) festgestellt. Finanziell besser gestellte Personen fordern demnach eine niedrigere Diskontrate. Laut Bocquého et al. (2013) sind die Diskontraten nicht von der Vermögenssituation abhängig, was auch unsere Ergebnisse zeigen. Dabei muss beachtet werden, dass die Vermögenssituation in der Literatur auf verschiedenen Wegen festgestellt wird. 


\section{Schlussfolgerung und Ausblick}

Die individuelle Zeitpräferenz stellt einen wesentlichen Einflussfaktor auf das Entscheidungsverhalten dar. Entscheidungen zum Umfang der Investitionen in Gebäude oder die Ausbildung, aber auch zum Umfang verschiedener Politikmassnahmen, wie der Wegekostenrechnung oder der Klimapolitik sind massgeblich durch die Zeitpräferenz (Diskontrate) bestimmt. Zur Diskontratenerfassung sind verschiedene Methoden verfügbar, von denen zwei experimentelle Ansätze in der Anwendung auf Landwirte verglichen werden. Dabei stellt die Methode nach Andersen et al. (2008) eine häufig angewendete, und die Methode nach Laury et al. (2012) eine neue und vereinfachende Erfassungsmethode, dar. Beide Methoden sollen keine signifikanten Unterschiede bei der Erfassung von Diskontraten aufweisen, was Laury et al. (2012) mit Studierenden zeigen. Da Ergebnisse von Studierenden nicht uneingeschränkt auf Unternehmer übertragen werden können, werden beide Methoden bei der Erfassung der Zeitpräferenz von Landwirten verglichen. Zudem werden die Methoden hinsichtlich ihrer Sensitivität bei den zur Erfassung verwendeten Geldbeträgen und die Methode nach Andersen et al. (2008) hinsichtlich der Ergebnisse bei explizit ausgewiesenen und nicht ausgewiesenen Zinssätzen im Experiment untersucht.

Es zeigt sich, dass sich die Methoden, entgegen den Ergebnissen von Laury et al. (2012), hinsichtlich der festgestellten Diskontraten bei Landwirten unterscheiden. Mit der Methode nach Andersen et al. (2008) erfasste Diskontraten sind niedriger als mit der Methode nach Laury et al. (2012) erfasste Diskontraten. Die Ergebnisse der Methode nach Andersen et al. (2008) sind sowohl hinsichtlich der verwendeten Geldbeträge zur Erfassung der Diskontraten als auch hinsichtlich des expliziten Ausweisens von Diskontraten sensitiv. Somit muss bei der Bestimmung der Diskontraten der verwendeten Erfassungsmethode, den zur Erfassung verwendeten Geldbeträgen und dem expliziten Ausweisen von Diskontraten grosse Aufmerksamkeit zukommen. Dabei sollte im Kontext der jeweiligen Fragestellung abgewogen werden, ob Zinssätze als entscheidungsunterstützende Information explizit ausgewiesen werden müssen oder nicht. Hinsichtlich der Anwendung beider Erfassungsmethoden auf landwirtschaftliche Unternehmer können folgende weitere Schlussfolgerungen gezogen werden: Bei der individuellen Diskontratenbestimmung muss für jede der beiden Methoden die Gruppe der inkonsistent entscheidenden Teilnehmer aus der Analyse ausgeschlossen werden. Im Falle von Landwirten ist die konsistente Gruppe signifikant jünger und hat mehr Bildungsjahre absolviert. Es stehen alternative Auswertungsmethoden, die inkonsistent entscheidende Teilnehmer in die Auswertung einschliessen (z.B. die Maximum-Likelihood Schätzung), zur Verfügung. Allerdings wird dabei die Zeitpräferenz von inkonsistent entscheidenden Personen, die die Aufgabenstellung eventuell nicht verstanden haben, möglicherweise mit relevanten Fehlern geschätzt. Somit sind die Ergebnisse einer Maximum-Likelihood Schätzung gerade bei signifikanten Unterschieden zwischen konsistent und inkonsistent entscheidenden Teilnehmern in ihrer Generalisierbarkeit mit Bedacht zu bewerten. Daraus lässt sich die Notwendigkeit einer vereinfachten Abfrage von Diskontraten oder einer adäquaten Bereinigungsmethode für inkonsistente Entscheider ableiten. Dieser Fragestellung sollte in zukünftigen Untersuchungen nachgegangen werden, um die experimentelle Diskontratenerfassung zu verbessern.

Die Methode nach Laury et al. (2012) vereinfacht die Erfassung und Ermittlung der Diskontraten für die Experimentteilnehmer und die Durchführenden des Experiments. Dem gegenüber liegen die Diskontraten, die mit der Methode nach Andersen et al. (2008) erfasst wurden näher am Opportunitätszins (einer Bankanlage). Dabei gilt es zu beachten, 
dass die Diskontraten in der Literatur durchaus unterschiedliche Höhen aufweisen. Im Rahmen der deutschen Wegekostenrechnung (Link et al. 2009) oder der klimapolitischen Bemühungen (Stern 2006) werden beispielsweise kleinere soziale Diskontraten verwendet, als sie sich aus experimentellen Untersuchungen ableiten lassen. In diesem Punkt sollte sich weitere Forschungsarbeit anschliessen, welche die Varianz der ermittelten Diskontraten untersucht, um mögliche Kalkulationsfehler bei der rechnerischen Verwendung der Diskontraten zu vermeiden.

Für Landwirte und politische Entscheidungsträger deuten die Ergebnisse an, dass Landwirte bei den von uns verwendeten intertemporalen Entscheidungssituationen verschiedene Diskontraten angeben. Aus diesem Grund sollten Landwirte selbst in jeder Situation bewusst über die getroffenen Entscheidungen nachdenken, um eine möglicherweise falsche zeitliche Allokation ihrer Ressourcen zu vermeiden. Politische Entscheidungsträger können die Ergebnisse als Anhaltspunkt verwenden, Landwirte hinsichtlich des Bewusstseins und der Einschätzung von Diskontraten zu unterstützen. Dadurch könnte möglicherweise die Einkommenssituation und der Strukturwandel in der Landwirtschaft positiv beeinflusst werden. Zudem wird die Politikfolgenabschätzung durch eine zutreffende Einschätzung der Diskontraten verbessert.

Für folgende Forschungsarbeiten wäre von Interesse zu untersuchen, wie sich bei der Anwendung der Methode nach Laury et al. (2012) ein explizites Ausweisen von Zinssätzen auf die angegebenen Diskontraten auswirkt. Um die hohe Inkonsistenzrate bei der Ermittlung der Risikoeinstellung bei der Methode nach Andersen et al. (2008) zu verringern, könnte für die Erfassung der Risikoeinstellung anstelle der HL-task eine alternative Vorgehensweise angewendet werden. Beispielsweise könnte die Methode von Eckel/Grossman (2008) Verwendung finden, bei der keine inkonsistenten Entscheidungen möglich sind, da nur eine präferierte Lotterie ausgewählt werden muss. Ausserdem sollte in weiterführenden Arbeiten die zeitliche Verzögerung zwischen den beiden Auszahlungszeitpunkten erhöht werden, um auf das Vorliegen von hyperbolischem Diskontieren zu prüfen. In weiteren Forschungsarbeiten sollten beide Methoden zur Zeitpräferenzmessung zudem mit Unternehmern anderer Sektoren und in anderen Ländern angewendet werden, um die Robustheit der gefundenen Ergebnisse zu überprüfen.

\section{Literaturverzeichnis}

Ablbrecht, M./Weber, M. (1997): An empirical study on intertemporal decision making under risk, in: Management Science, Vol. 43, S. 813-826.

Alfen Consult/AVISO/IVM (2014): Berechnung der Wegekosten für das Bundesfernstraßennetz sowie der externen Kosten nach Maßgabe der Richtlinie 1999/62/EG für die Jahre 2013 bis 2017, Weimar.

Anderhub, V., et al. (2001): On the interaction of risk and time preferences: an experimental study, in: German Economic Review, Vol. 2, S. 239-253.

Andersen, S., et al. (2014): Discounting behavior: A reconsideration, in: European Economic Review, Vol. 71, S. 15-33.

Andersen, S., et al. (2010): Preference heterogeneity in experiments: comparing the field and laboratory, in: Journal of Economic Behavior and Organization, Vol. 73, S. 209-224.

Andersen, S., et al. (2008): Eliciting risk and time preferences, in: Econometrica, Vol. 76, S. 583618. 
Andersen, S., et al. (2006): Elicitation using multiple price list formats, in: Experimental Economics, Vol. 9, S. 383-405.

Anderson, L.R./Stafford, S.L. (2009): Individual decision-making experiments with risk and intertemporal choice, in: Journal of Risk and Uncertainty, Vol. 38, S. 51-72.

Andreoni, J./Sprenger, C. (2012): Estimating time preferences from convex budgets, in: American Economic Review, Vol. 102, S. 3333-3356.

Barr, S.H./Hitt, M.A. (1986): A comparison of selection decision models in manager versus student models, in: Personnel Psychology, Vol. 39, S. 599-617.

Beckers, T., et al. (2009): Zeitliche Homogenisierung und Berücksichtigung von Risiko im Rahmen von Wirtschaftlichkeitsuntersuchungen, Studie im Auftrag des Bundesrechnungshofes, Berlin und Speyer.

Benhabib, J., et al. (2010): Present-bias, quasi-hyperbolic discounting, and fixed costs, in: Games and Economic Behavior, Vol. 69, S. 205-223.

Benzion, U., et al. (1989): Discount rates inferred from decisions: an experimental study, in: Management Science, Vol. 35, S. 270-284.

Bocquého, G., et al. (2013): Reversal and magnitude effects in long-term time preferences: results from a field experiment, in: Economics Letters, Vol. 120, S. 108-111.

Chabris, C., et al. (2008): Individual laboratory-measured discount rates predict field behavior, in: Journal of Risk and Uncertainty, Vol. 37, S. 237-269.

Coble, K.H./Lusk, J.L. (2010): At the nexus of risk and time preferences: An experimental investigation, in: Journal of Risk and Uncertainty, Vol. 41, S. 67-79.

Coller, M./Williams, M.B. (1999): Eliciting individual discount rates, in: Experimental Economics, Vol. 2, S. 107-127.

Duquette, E., et al. (2012): Farmer discount rates: experimental evidence, in: American Journal of Agricultural Economics, Vol. 94, S. 451-456.

Eckel, C.C./Grossman, P.J. (2008): Forecasting risk attitudes: an experimental study using actual and forecast gamble choices, in: Journal of Economic Behavior and Organization, Vol. 68, S. 117.

Fabrmeir, L., et al. (2013): Regression: Models, methods and applications, 1. Aufl. Berlin 2013.

Frederick, S. (2003): Time preference and personal identity, in: Loewenstein, George., Read, Daniel/ Baumeister, Roy F. (Hrsg.): Time and decision. New York 2003, S. 89-113.

Frederick, S., et al. (2002): Time discounting and time preference: a critical review, in: American Economic Review, Vol. 40, S. 351-401.

Friedl, B., et al. (2014): Gesamtwirtschaftliche Bewertung von Smart-Grids-Lösungen anhand einer KostenNutzen-Analyse, 13. Symposium Energieinnovation an der TU Graz. Graz.

Gollier, C./Zeckhauser, R. (2005): Aggregation of heterogeneous time preferences, in: Journal of Political Economy, Vol. 113, S. 878-896.

Harrison, G.W., et al. (2009): Risk attitudes, randomization to treatment, and self-selection into experiments, in: Journal of Economic Behavior and Organization, Vol. 70, S. 498-507.

Harrison, G.W., et al. (2007): Estimating risk attitude in Denmark: A field experiment, in: Scandinavian Journal of Economics, Vol. 109, S. 341-368.

Harrison, G.W., et al. (2002): Estimating individual discount rates in Denmark: a field experiment, in: American Economic Review, Vol. 92, S. 1606-1617. 
Harrison, G.W./List, J.A. (2008): Naturally occurring markets and exogenous laboratory experiments: a case study of the winner's curse, in: The Economic Journal, Vol. 118, S. 822-843.

Hausman, J.A. (1979): Individual discount rates and the purchase and utilization of energy using durables, in: Bell Journal of Economics, Vol. 10, S. 33-54.

Herberich, D.H./List, J.A. (2012): Digging into background risk: experiments with farmers and students, in: American Journal of Agricultural Economics, Vol. 94, S. 457-463.

Hirschauer, N., et al. (2014): Eliciting risk attitudes- how to avoid mean and variance bias in Holtand-Laury lotteries, in: Applied Economics Letters, Vol. 21, S. 35-38.

Holt, C.A./Laury, S.K. (2002): Risk aversion and incentive effects, in: American Economic Review, Vol. 92, S. 1644-1655.

Jacobson, S./Petrie, R. (2009): Learning from mistakes: what do inconsistent choices over risk tell us? In: Journal of Risk and Uncertainty, Vol. 38, S. 143-158.

Khera, I.P./Benson, J.D. (1970): Are students really poor substitutes for businessmen in behavioral research? In: Journal of Marketing Research, Vol. 7, S. 529-532.

Lambson, V.E./Jensen, F.E. (1995): Sunk costs and the variability of firm value over time, in: The Review of Economics and Statistics, Vol. 77, S. 535-544.

Laury, S.K., et al. (2012): Avoiding the curves: direct elicitation of time preferences, in: Journal of Risk and Uncertainty, Vol. 44, S. 181-217.

Lence, S.H. (2000): Using consumption and asset return data to estimate farmers' time preference and risk attitudes, in: American Journal of Agricultural Economics, Vol. 82, S. 934-947.

Link, H., et al. (2009): Wegekosten und Wegekostendeckung des Straßen- und Schienenverkehrs in Deutschland im Jahre 2007. Forschungsprojekt der DIW Berlin, Abteilung Energie, Verkehr, Umwelt im Auftrag des BGL, ADAC und BDI.

Maart-Noelck, S.C./Mußhoff, O. (2014): Measuring the risk attitude of decision-makers: are there differences between groups of methods and persons? In: Australian Journal of Agricultural and Resource Economics, Vol. 58, S. 336-352.

Moore, M.J./Viscusi W.K. (1990): Models for Estimating Discount Rates for Long-Term Health Risks Using Labor Market Data, in: Journal of Risk and Uncertainty, Vol. 3, S. 381-401.

Moschini, G./Hennessy, D.A. (2001): Uncertainty, risk aversion, and risk management for agricultural producers, in: Gardner, Bruce L./Rausser, Gordon C. (Hrsg.). Handbook of Agricultural Economics: Agricultural Production 1A, 1. Aufl. Amsterdam 2001.

OECD (Organisation for Economic Co-operation and Development) (1999): Classifying Educational Programmes: Manual for ISCED-97 Implementation in OECD Countries.

Pender, J.L. (1996): Discount rates and credit markets: theory and evidence from rural India, in: Journal of Development Economics, Vol. 50, S. 257-296.

Reynaud, A./Couture, S. (2012): Stability of risk preference measures: results from a field experiment on French farmers, in: Theory and Decision Vol. 73, S. 203-221.

Roe, B.E./Just, D.R. (2009): Internal and external validity in economics research: tradeoffs between experiments, field experiments, natural experiments, and field data, in: American Journal of Agricultural Economics, Vol. 91, S. 1266-1271.

Smith, V.L. (1982): Microeconomic systems as an experimental science, in: American Economic Review, Vol. 72, S. 923-955.

Statistisches Bundesamt (Hrsg.) (2011): Wirtschaftsrechnungen: Einkommens- und Verbrauchsstichprobe: Aufwendungen privater Haushalte für Nahrungsmittel, Getränke und Tabakwaren 2008. Fachserie 15 (3), Wiesbaden. 
Stern, N. (2006): Stern Review Report on the Economics of Climate Change. Cambridge University Press, Cambridge

Tanaka, T, et al. (2010): Risk and time preferences: linking experimental and household survey data from Vietnam, in: American Economic Review, Vol. 100, S. 557-571.

Thaler, R. (1981): Some empirical evidence on dynamic inconsistency, in: Economics Letters, Vol. 8, S. 201-207.

Train, K. (1985): Discount rates in consumers' energy-related decisions: a review of the literature, in: Energy, Vol. 10, S. 1243-1253.

Daniel Hermann, M. Sc., ist wissenschaftlicher Mitarbeiter am Lehrstuhl für Landwirtschaftliche Betriebslehre der Georg-August-Universität Göttingen.

Anschrift: Universität Göttingen, Department für Agrarökonomie und Rurale Entwicklung, Platz der Göttinger Sieben 5, D-37073 Göttingen, Tel.: +49 (0)551 399321, Fax: +49 (0)551 3922030, E-Mail:dherman@gwdg.de

Dörthe Rüther, M. Sc., war wissenschaftliche Mitarbeiterin am Lehrstuhl für Landwirtschaftliche Betriebslehre der Georg-August-Universität Göttingen.

Anschrift: Universität Göttingen, Department für Agrarökonomie und Rurale Entwicklung, Platz der Göttinger Sieben 5, D-37073 Göttingen, Tel.: +49 (0)551 399321, Fax: $+49(0) 5513922030$

Oliver Mußhoff, Dr., ist Professor für Landwirtschaftliche Betriebslehre an der Georg-August-Universität Göttingen.

Anschrift: Universität Göttingen, Department für Agrarökonomie und Rurale Entwicklung, Platz der Göttinger Sieben 5, D-37073 Göttingen, Tel.: +49 (0)551 394842, Fax: +49 (0)5513922030, E-Mail: oliver.musshoff@agr.uni-goettingen.de 